\title{
RESEARCH
}

Open Access

\section{Evolution of pogo, a separate superfamily of IS630-TC1-mariner transposons, revealing recurrent domestication events in vertebrates}

Bo Gao, Yali Wang, Mohamed Diaby, Wencheng Zong, Dan Shen, Saisai Wang, Cai Chen, Xiaoyan Wang and Chengyi Song ${ }^{*}$ (D)

\begin{abstract}
s
Background: Tc1/mariner and Zator, as two superfamilies of IS630-TC1-mariner (ITm) group, have been well-defined. However, the molecular evolution and domestication of pogo transposons, once designated as an important family of the TC1/mariner superfamily, are still poorly understood.

Results: Here, phylogenetic analysis show that pogo transposases, together with Tc1/mariner, DD34E/Gambol, and Zator transposases form four distinct monophyletic clades with high bootstrap supports ( $>=74 \%$ ), suggesting that they are separate superfamilies of $/ \mathrm{Tm}$ group. The pogo superfamily represents high diversity with six distinct families (Passer, Tigger, pogoR, Lemi, Mover, and Fot/Fot-like) and wide distribution with an expansion spanning across all the kingdoms of eukaryotes. It shows widespread occurrences in animals and fungi, but restricted taxonomic distribution in land plants. It has invaded almost all lineages of animals - even mammals_-and has been domesticated repeatedly in vertebrates, with 12 genes, including centromere-associated protein B (CENPB), CENPB DNA-binding domain containing 1 (CENPBD1), Jrk helix-turn-helix protein (JRK), JRK like (JRKL), pogo transposable element derived with KRAB domain (POGK), and with ZNF domain (POGZ), and Tigger transposable element-derived 2 to 7 (TIGD2-7), deduced as originating from this superfamily. Two of them (JRKL and TIGD2) seem to have been co-domesticated, and the others represent independent domestication events. Four genes (TIGD3, TIGD4, TIGD5, and POGZ) tend to represent ancient domestications in vertebrates, while the others only emerge in mammals and seem to be domesticated recently. Significant structural variations including target site duplication (TSD) types and the DDE triad signatures (DD29-56D) were observed for pogo transposons. Most domesticated genes are derived from the complete transposase genes; but CENPB, POGK, and POGZ are chimeric genes fused with additional functional domains.

Conclusions: This is the first report to systematically reveal the evolutionary profiles of the pogo transposons, suggesting that pogo and Tc1/Mariner are two separate superfamilies of ITm group, and demonstrating the repeated domestications of pogo in vertebrates. These data indicate that pogo transposons have played important roles in shaping the genome and gene evolution of fungi and animals. This study expands our understanding of the diversity of pogo transposons and updates the classification of ITm group.
\end{abstract}

Keywords: pogo, DNA transposons, Tc1/mariner, IS630, Evolution, Domestication

\footnotetext{
* Correspondence: cysong@yzu.edu.cn

College of Animal Science and Technology, Yangzhou University, Yangzhou 225009, Jiangsu, China
} 


\section{Introduction}

Transposable elements or transposons are viewed as molecular parasites and segments of genetic material that can ensure their own replication (albeit with the help of host factors). They are sometimes called "jumping genes" for their ability to jump around from place to place on chromosomes and are found in both prokaryotic and eukaryotic genomes [1, 2]. Based on the "jumping" mechanism, transposons are classified into RNA transposons (retrotransposons), which move using an RNA intermediate, along with reverse transcriptase to produce the complementary DNA, and DNA transposons, which move about using a DNA intermediate associated with a transposase [3]. Multiple transposition mechanisms of DNA transposons have been defined and they can be subdivided into three major types: the cutand-paste, peel-and-paste, and self-synthesizing transposons [3-5].

Transposons are thought to have played important roles in the evolution of individual genes and in shaping the genomic landscape of their host $[6,7]$. It has been suggested that transposons play important roles in genome size variations in vertebrates [8,9], and they constitute a large fraction (30-50\%) of mammal genomes [10]. It has been found that some DNA transposons can undergo "molecular domestication" a process through which they evolve new cellular functions but also lose their mobility due to loss of function of the two minimally required functional components: the terminal inverted repeat (TIR) sequences and the transposase [7, 11]. Many protein-coding genes in mammals have evolved from DNA transposons; about 50 domesticated genes in the human genome have been reported [7, 11], and these have derived from diverse DNA transposons, such as THAP9 derived from the $P$ element [12], SETMAR derived from Tc1/mariner [13], RAG proteins derived from Transib [14], and PGBD5 derived from piggyBac [15].

Tc1/mariner, a superfamily of cut-and-paste transposons named after the first element identified in Caenorhabditis elegans (Transposon C. elegans number 1, Tc1) [16] and Drosophila mauritiana (mariner) [17] is thought to be the most widespread group of DNA transposons, and multiple distinct families (DD34D/mariner, DD37D/maT, DD39D, DD41D, DD34E/Tc1, DD35E/TR, DD36E/IC, and DD37E/TRT) of Tc1/mariner have been well-defined [18-23]. The eukaryotic superfamily Tc1/ mariner is related to the bacterial IS630 family [24], which is also referred to as the IS630-Tc1-Mariner (ITm) group [25-27]. Zator was identified as a superfamily and related to the bacterial TP36 family of transposases [28]. However, Zator and TP36 are also clustered with the bacterial IS630 family, along with the Tc1/mariner [28], indicating that the ITm group represents high diversity and the phylogenetic relationship across these transposons is still waiting to be defined. The pogo element was firstly identified in flies [29], then diverse relative transposons including Tigger in humans [30], Fot, Tan1, Pot1, Pot2, Flipper, and Aft1transposons in fungi [31-36], pogo-like elements (Lemi1) in plants [37], and pogo-like elements in teleosts [38] have been identified and they were close to pogo transposase in phylogenetic position $[9,26,38]$. This group was named as $\mathrm{DD} \times \mathrm{D} /$ pogo [26], and it was believed to belong to the Tc1/mariner superfamily for long time [25, 26]. However, the origin, taxonomic distribution, diversity, and molecular domestication of the pogo transposons remain largely unknown. In addition, although the domestication of CENPB has been well characterized [39], the origins of several other related genes, including TIGD1-TIGD7, JRK and JRKL, are ambiguous [39, 40], and the evolutionary relationships between them remain unknown. Here, we systematically investigate the taxonomic distribution of pogo transposons, as well as their domestication in vertebrates, and characterize the phylogenetic relationships, structural organization, and conservation of these transposons and their domesticated proteins. Our data display, for the first time, the entire evolutionary landscape of pogo transposons and their domestication in vertebrates, and we also provide evidence to support that pogo is a separate superfamily and evolved independently from IS630 transposases. These findings have important implications for understanding the evolution of the pogo transposons, as well as their impact on genome and gene evolution.

\section{Results}

\section{pogo and Tc1/Mariner are two distinct superfamilies of ITm transposons}

To define the phylogenetic position of pogo transposons, here we retrieved all bacteria IS630 transposase sequences (121 sequences) containing DDE domains from ISfinder database [41], classified them into 11 clades by using the IS256 transposase as outgroup (Additional file 1: Fig. S1). Then, 19 representative IS630 transposase sequences including the 11 clades, Tc1/mariner transposase families identified previously [21-23, 26, 42-47], and Zator transposases, which were defined as a superfamily close to ITm group [28], were combined with all identified pogo transposases to infer a phylogenetic tree by using maximum likelihood methods with the IQTREE program [48]. The resulting tree shows that although pogo, Tc1/mariner (including DD34E/Tc1, DD35E/TR, DD36E/IC, DD34D/mariner, DD37D/maT, DD37E/TRT, DD37D, DD39D, and DD41D), Zator, and DD34E/Gambol transposases are sister clades, they formed four distinct highly supported monophyletic clades, with 74, 99, 92, and 100\% bootstrap supports for 
pogo, Zator, Tc1/mariner, and DD34E/Gambol clades respectively (Fig. 1a and Additional file 2: Fig. S2). Therefore, we assume that pogo, Tc1/mariner, DD34E/ Gambol, and Zator transposons may have evolved independently from IS630 transposons and form separate superfamilies of eukaryotic DNA transposons. In order to investigate the origin of pogo transposons, we also conducted Blast searches against the bacteria genomes. However, we could not identify any other insertion sequences homologous to pogo transposons, beside IS630
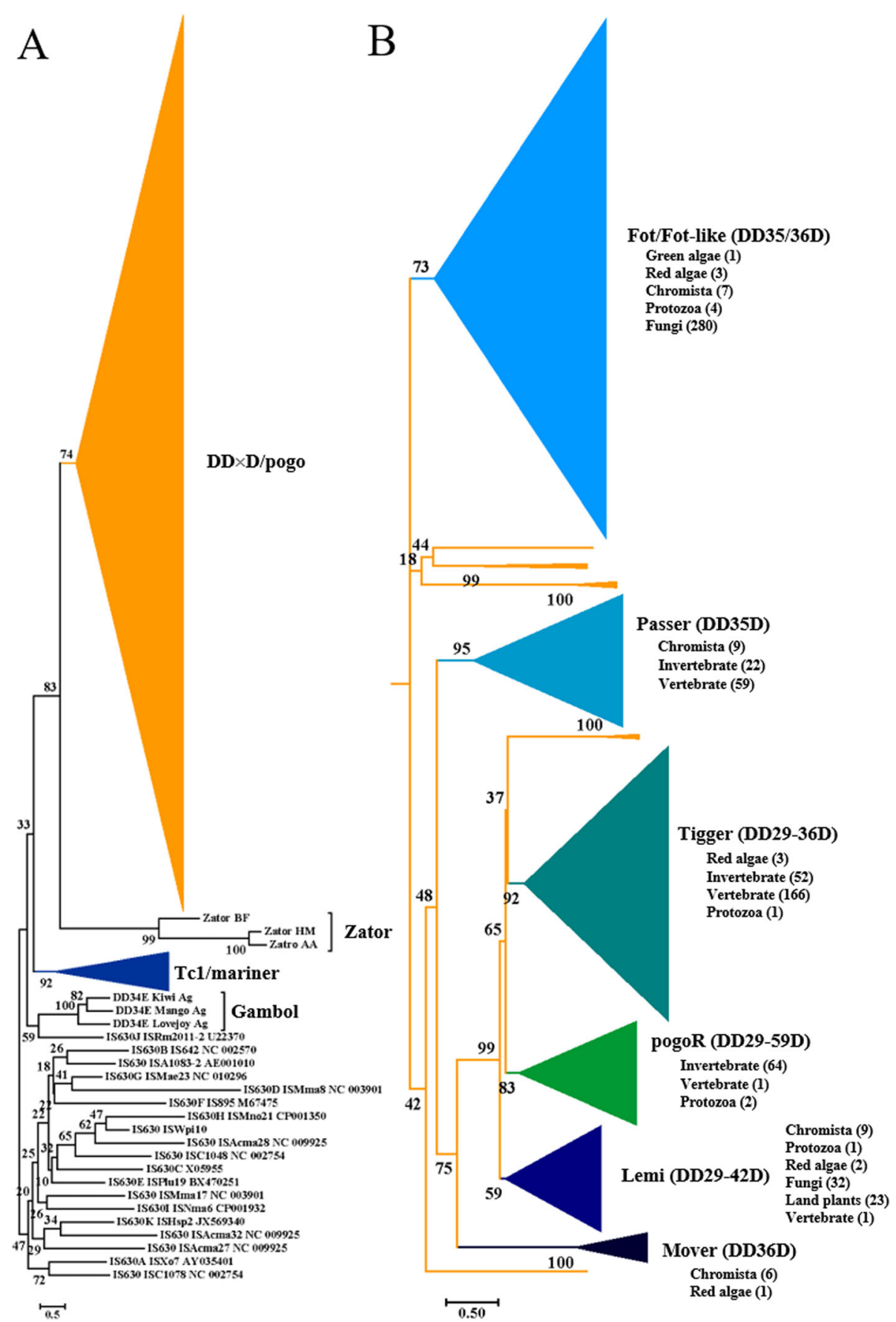

Fig. 1 Phylogenetic tree of pogo transposases. a Unrooted phylogenetic tree of pogo transposases relative to Zator, IS630, DD34E/Gambol, and previously described TC1/mariner families. The unrooted phylogenetic tree was inferred using the maximum likelihood method with the IQ-TREE program (see Additional file 2: Fig. S2 for an uncollapsed tree). Transposase sequences of DD36E/IC, DD35E/TR, DD37E/TRT are from the references $[22,23,42]$. GenBank access numbers of other reference elements are included in Additional file 2: Fig. S2. The number of species/ organisms containing pogo elements for each pogo family is given in brackets for each lineage or group of eukaryotes 
elements, indicating that pogo may still originate from the insertion sequences of IS630 group. The pogo transposons were further classified into six main families (Passer, Tigger, pogoR, Lemi, Mover, and Fot/Fot-like). Four of them (Fot/Fot-like [49], Tigger [30] pogoR [29], and Lemi [37]) correspond to the known families reported previously, and have $73,92,83$, and $59 \%$ bootstrap supports respectively, pogoR is the first pogo transposon identified in fly (Drosophila melanogaster) [29], while both Passer and Mover have been defined as new families with 95 and 100\% bootstrap supports respectively (Fig. 1b). The main pogo superfamily also consists of a well-supported grouping including diverse minor clades (Fig. 1b and Additional file 2: Fig. S2).

\section{Extensive distribution of pogo transposons in eukaryotes}

The species in which pogo transposons were detected, their classification, structural characteristics, sequences and genome coordinates in each genome were listed in Additional file 3: Table S1. The pogo superfamily is absent from prokaryotes, but present in all kingdoms of eukaryotes, including plants (red algae, green algae, and land plants), Chromista (Stramenopiles and Rhizaria), protozoa (Amoebozoa, Excavata, Choanoflagellata, and Ichthyosporea), fungi, and animals. This superfamily is also distributed widely within the phyla and classes of invertebrates and vertebrates, only being absent from the Ctenophora and Cephalochordata of invertebrates and Caudata of vertebrates (Fig. 2).

Although three families (pogoR, Passer, and Tigger) are mainly present in the animal kingdom, only Passer and Tigger transposons displayed extensive distributions in vertebrates (Fig. 2). The pogoR transposons are mainly distributed within the invertebrates (64 species), including the Cnidaria, Echinodermata, Platyhelminthes, Nematoda, Arthropoda, and Mollusca, and only present in one species (Latimeria chalumnae/lobe-finned fish) of vertebrates (Figs. 1 and 2, and Additional file 3: Table S1). Passer forms a big family distributed in all detected phyla of invertebrates (22 species) except for the Ctenophora and Cephalochordata, most classes (ray-finned fish, lobe-finned fish, amphibians, reptiles, and mammals) of vertebrates (59 species), and nine species (Stramenopiles) of Chromista. Within mammals, Passer transposons are only found in two orders of eutherians (Chiroptera and Afrotheria) (Fig. 1 and Fig. 2, and Additional file 3: Table S1). Tigger also comprises a big family that was first reported in humans [30], and is distributed across most phyla of invertebrates (Porifera, Cnidaria, Platyhelminthes, Nematoda, Arthropoda, and Mollusca) (52 species) and all the classes of vertebrates (166 species), except for the Caudata. Some elements in three species of red algae are also defined as Tigger (Fig. 1 and Fig. 2, and Additional file 3: Table S1).
Furthermore, Tigger transposons display extensive distribution within most orders of eutherian mammals (Fig. 2). We even identified Tigger transposons in most species of primates (Additional file 3: Table S1). However, the taxonomic distribution of these families including Tigger may be underestimated due to the exclusion of the truncated elements of ancient copies.

Lemi transposons are present in red algae (two species), land plants (23 species), Chromista (nine species), protozoa (one species), fungi (32 species), and animals (one species of lobe-finned fish), while Mover forms a small clade and displays a restricted distribution within red algae (one species) and Chromista (five species of Stramenopiles and one species of Rhizaria) (Fig. 1 and Fig. 2, and Additional file 3: Table S1). By contrast, Fot/ Fot-like is a very large family, which also consists in multiple minor clades with varying bootstrap support (Fot-like elements) that share a sister-group relationship with a well-supported (100\%) clade of the Fot family (Additional file 2: Fig. S2). Fot is distributed extensively in fungi (280 species), while Fot-like elements are distributed within the Chromista (seven species of Stramenopiles), protozoa (each species in each of the Amoebozoa, Excavata, Choanoflagellata, and Ichthyosporea), one species of green algae and three species of red algae (Figs. 1 and 2, and Additional file 3: Table S1).

\section{Wide occurrence of pogo transposons in fungi}

The pogo transposons were detected within most subgroups of plants, including red and green algae and land plants (Fig. 2). They did not undergo significant amplification among land plants, in which only one small clade of pogo transposons (named Lemi) was identified in 23 Eudicot species (one species in the Ranunculales, 16 Rosid species, and six Asterid species) (Fig. 1 and Fig. 3a, and Additional file 4: Fig. S3A and Additional file 3: Table S1). By contrast, wide distribution of pogo transposons was observed in fungi. One was defined as the Lemi family, distributed among two species of Saccharomycotina, and 30 species across four classes of Pezizomycotina (Eurotiomycetes, Dothideomycetes, Leotiomycetes, and Sordariomycetes; Fig. 1 and Fig. 3b, Additional file 3: Table S1 and Additional file 4: Fig. S3A). The other was defined as the Fot family, further classified into four distinctive clades $(F o t A-D)$, which displayed an extensive distribution in fungi; these were detected in $82,206,27$, and 57 species, respectively, across six classes of Pezizomycotina/Ascomycota (Eurotiomycetes, Dothideomycetes, Lecanoromycetes, Leotiomycetes, Sordariomycetes, and Pezizomycetes) and two classes of Basidiomycota (Fig. 3b-c, Additional file 3: Table S1, and Additional file 4: Fig. S3B). In addition, the copy number of Fot elements in the genomes of different fungi species varies dramatically, from only one copy ( $>90 \%$ of identity and $>1000$ 


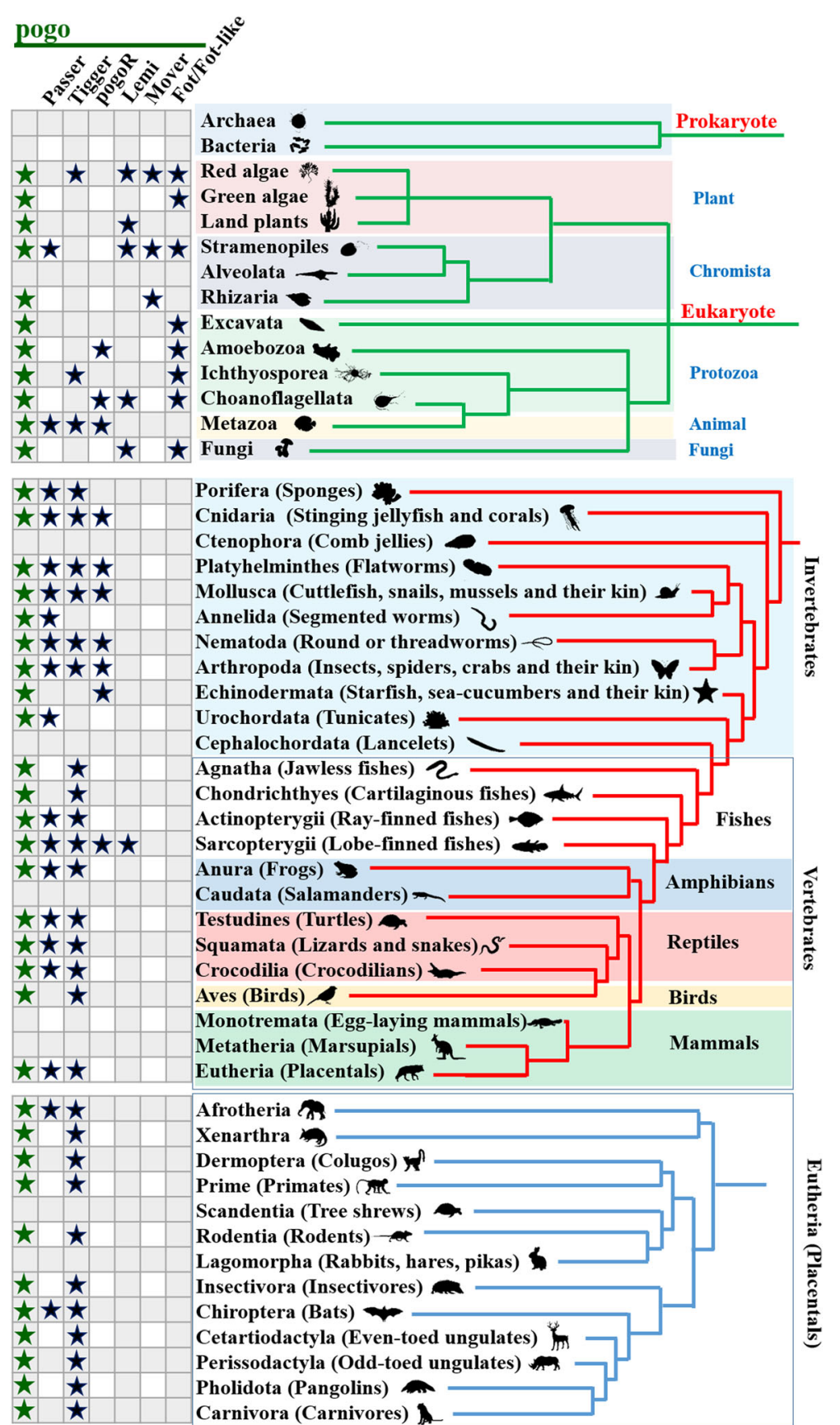

Fig. 2 Distribution of pogo transposons. Symbols in green represent the distribution of all pogo transposons including Passer, pogoR, Tigger, Lemi, Mover, and the unclassified elements

bp in length) to over hundred copies, but most of them have less than 200 copies (Additional file 3: Table S1).

\section{Significant structural variations of pogo transposons}

The members of pogo superfamily have a total length range of 1.20-5.20 kilobases (kb), with one or two open reading frames (ORFs) encoding transposases of 300977 amino acids (aa), flanked by TIRs and TSDs (Fig. 4). Significant structural variations were observed for pogo transposons. Two new types of TSDs (TAA and TAAA) were identified in Fot and Fot-like transposons, respectively, which differed from the classical 2 bp TA-flanked TSD of Tc1/mariner [52], while other pogo transposon families were still characterized by classical TA TSDs. Most Fot transposons (341 out of 385 elements) have TAA TSDs, while the rest Fot elements (54 out of 385 elements) have TA TSDs, which only present in FotC and FotD clades. Ten Fot-like elements have TAAA 
A

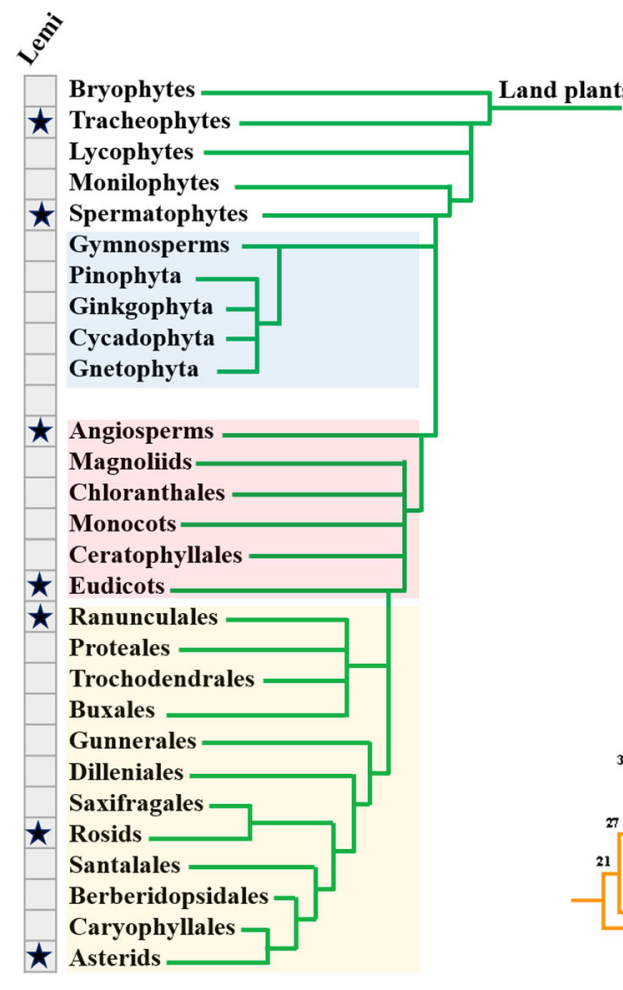

C

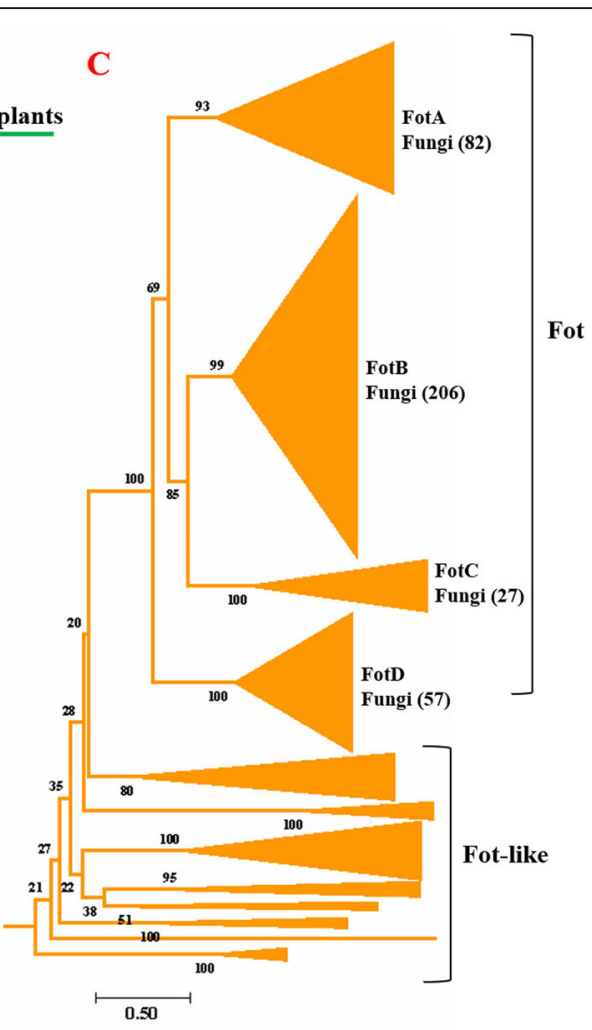

ot

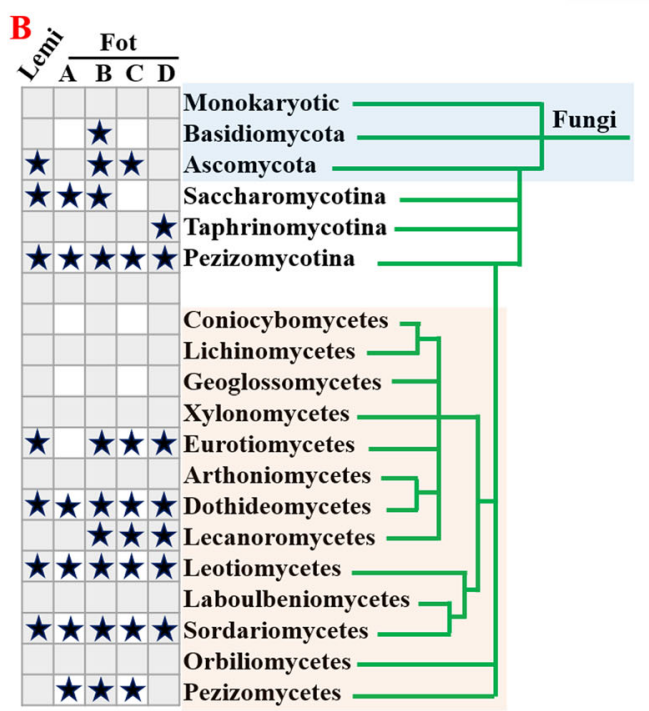

ungi

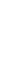




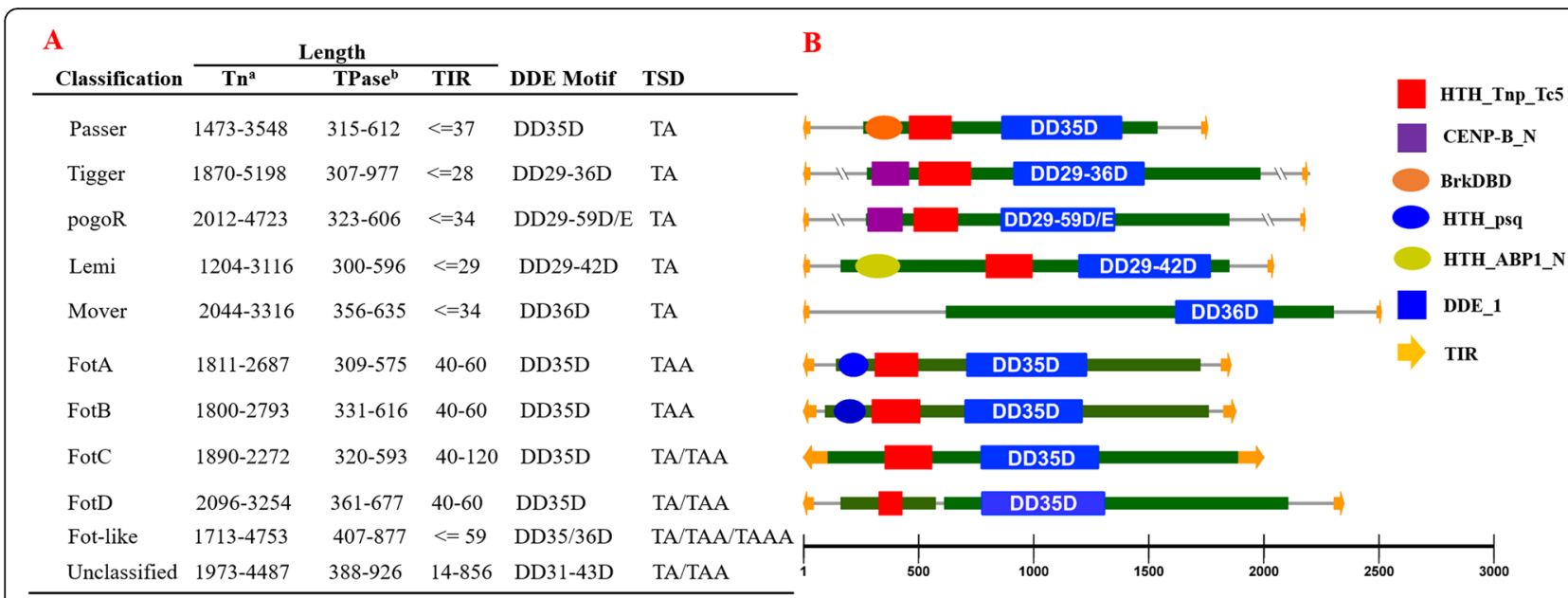

Fig. 4 Structural organization of pogo transposons. a Summary of structural organization of pogo transposons. b Schematic of structural organization correspondence to each pogo transposon family/calde. The domain architecture of transposases was screened using hmmscan [50]; the names of DBD and DDE motifs have been designated according to the Pfam database [51]. ${ }^{\text {TT }}$ The lengths of full transposons with detectable TIR and TSD sequences; ${ }^{\text {}}$ The length of a putatively intact transposase (TPase) (> 300 aa) in a full-length transposon

36D), pogoR (DD29-59D/E), and Lemi (DD29-42D). This number was highly conserved in most other Tc1/ mariner families [26, 38, 42], while almost all Passer and Fot/Fot-like transposons were characterized by DD35D, and all Mover transposons by DD36D. Only four Fot-like transposons in Ichthyosporea are characterized by a DD36D domain. Furthermore, very large spacings (DD56D and DD59D) were observed for some pogoR transposons in Amoebozoa (Fig. 1 and Fig. 4, and Additional file 3: Table S1). Five types of DNA binding domain (DBD) motifs, designated CENP-B_N, HTH ABP1_N, HTH_Tnp_Tc5, BrkDBD, and HTH_psq in the Pfam database [51], were identified in N-terminals of pogo transposases. Fot and Fot-like transposases harbored two types of motifs (HTH_psq and HTH_Tnp Tc5), or a single motif of HTH_psq or HTH_Tnp_Tc5 in the DBD domain, while the Passer transposases harbored double DBD motifs of BrkDBD and $\mathrm{HTH}_{-}$ Tnp_Tc5, or a single DBD motif of HTH_Tnp_Tc5. Lemi transposases are characterized by a single $\mathrm{DBD}$ motif (HTH_Tnp_Tc5), or double DBD motifs of HTH ABP1_N and HTH_Tnp_Tc5. pogoR and Tigger transposases harbor double DBD motifs of CENP-B_N and HTH_Tnp_Tc5, or a single DBD motif of HTH_Tnp_ Tc5. The DBD motif of Mover transposases, taxonomically restricted to red algae, and Chromista, was not detectable by hmmscan (Fig. 4 and Additional file 3: Table S1).

\section{Recurrent domestication events of pogo transposons in vertebrates}

Based on an analysis of the RefSeq Representative Genome Database, we found that pogo transposons underwent recurrent domestication in vertebrates. Over
1500 protein sequences derived from pogo transposases were found, representing at least 12 well-annotated genes (Additional file 5: Table S2), including CENPBD1, JRK, JRKL, TIGD2-7, POGK and POGZ, beside the CENPB, which has been characterized previously [39]. The phylogenetic tree revealed that these protein sequences were derived from three families (Passer, pogoR, and Tigger) of pogo transposases, and can be classified into three groups: Group I, which includes four genes (TIGD3, TIGD4, TIGD6, and CENPB) derived from pogoR transposase; Group II, which includes five genes (CENPBD1, JRK, JRKL, TIGD2, TIGD5, and TIGD7) derived from Tigger transposase; and Group III, which includes POGK and POGZ derived from Passer transposase (Fig. 5a, Additional file 6: Fig. S4 and Additional file 7: Fig. S5). The continuous phylogenetic distribution of these genes, coupled with high sequence identity ( $>74 \%$ ) and low nonsynonymous to synonymous substitutions $(\mathrm{Ka} / \mathrm{Ks})$ ratios $(<1)$ (Z-test, $P<0.05)$ (Fig. $5 \mathrm{~b}$ and Table 1), which provides a measure of selection acting to maintain amino acid sequence [53], strongly suggests that they evolved under strong purifying (negative) selection, and tend to represent stationary domesticated genes. In addition, we also identified many TIGD1s and TIGD1-like sequences (TIGD1Ls), which are homologous to Tigger transposases; however, all TIGD1s and most TIGD1Ls are present as multiple copies. The TIGD1Ls were grouped into several small clades displaying low sequence identities and very narrow distribution among taxa (data not shown), indicating that they are akin to pseudogenes, and therefore excluded from this analysis.

Based on the phylogenetic analysis and the $\mathrm{Ka} / \mathrm{Ks}$ ratio analysis, the stationary domesticated genes were 


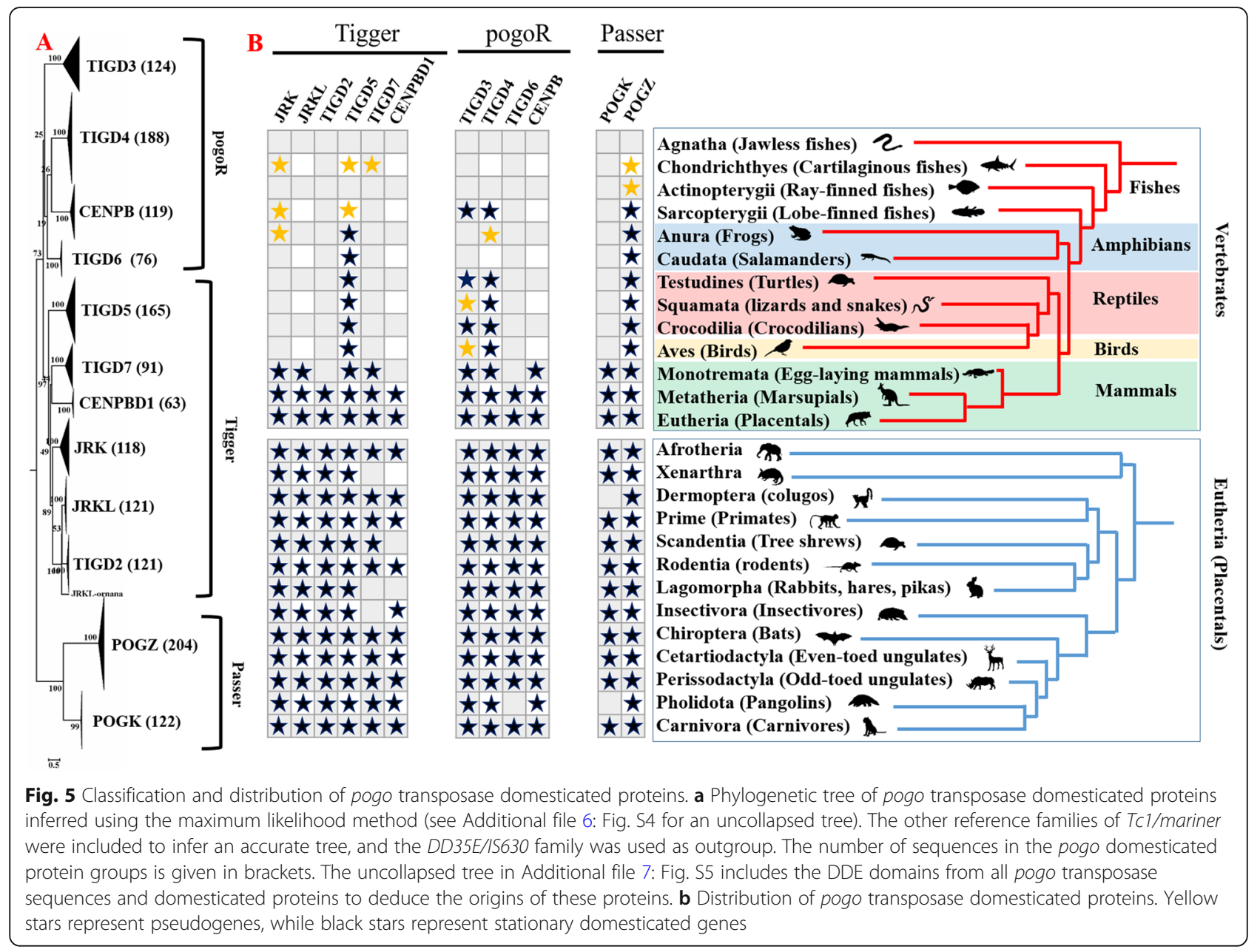

confirmed, and their taxonomic distribution was summarized in Fig. $5 \mathrm{a}$ and b. Together, these data revealed four genes (TIGD3, TIGD4, TIGD5, and POGZ) tend to represent ancient domestication events in vertebrates, while eight (JRK, JRKL, TIGD2, CENPB, CENPBD1, TIGD6, TIGD7, and POGK) appear to be present as recent domestication events in mammals (Fig. 5b). TIGD3 and TIGD4 display continuous distributions in mammals, but have a more uneven phyletic distribution in non-mammalian vertebrates. TIGD3, TIGD4, and POGZ were likely recruited to the Sarcopterygii/lobe-finned fish superclass, prior to the split of Amniota and Amphibia, but TIGD3 was subsequently lost from the Anura, Caudata, Squamata, and Aves, while TIGD4 was lost from the Anura and Caudata (Fig. 5b). Pseudogenes of TIGD3 were detected in Squamata and Aves, those of TIGD4 were detected in Anura, which are phylogenetically close to the stationary domesticated genes, but forming a distinct clade and displaying low sequence identity within clades (Additional file 5: Table S2 and Additional file 7: Fig. S5). TIGD5 has emerged in Amniota and Amphibia, and both TIGD5 and POGZ display continuous distributions and seem to have been maintained in most lineages of vertebrates after domestication. CENPB, JRK, JRKL, TIGD7, and POGK might have originated in the egg-laying mammals (Monotremata), prior to the divergence of the marsupials and eutherian ("placental") groups, while TIGD2, TIGD6, and CENPBD1 seem to have emerged in the Theria. All these genes display continuous distributions in mammals except for CENPBD1, which is absent from the Lagomorpha, Scandentia, and Xenarthra, and missing from most species of primates and rodents, but has continuous distribution in the Laurasiatheria (Chiroptera, Cetartiodactyla, Perissodactyla, Pholidota, and Carnivora; Fig. $5 \mathrm{~b}$ and Additional file 5: Table S2), suggesting that it might be a very recent domestication event in mammals. Pseudogenes of these genes were also detected: JRK in cartilaginous fish and lobe-finned fish, and Anura; TIGD7 in cartilaginous fish; TIGD5 in cartilaginous and lobe-finned fish; and POGZ in cartilaginous and ray-finned fish (Fig. 5b, Additional file 5: Table S2 and Additional file 7: Fig. S5). In addition, the phylogenetic tree also suggests that most genes arose by 


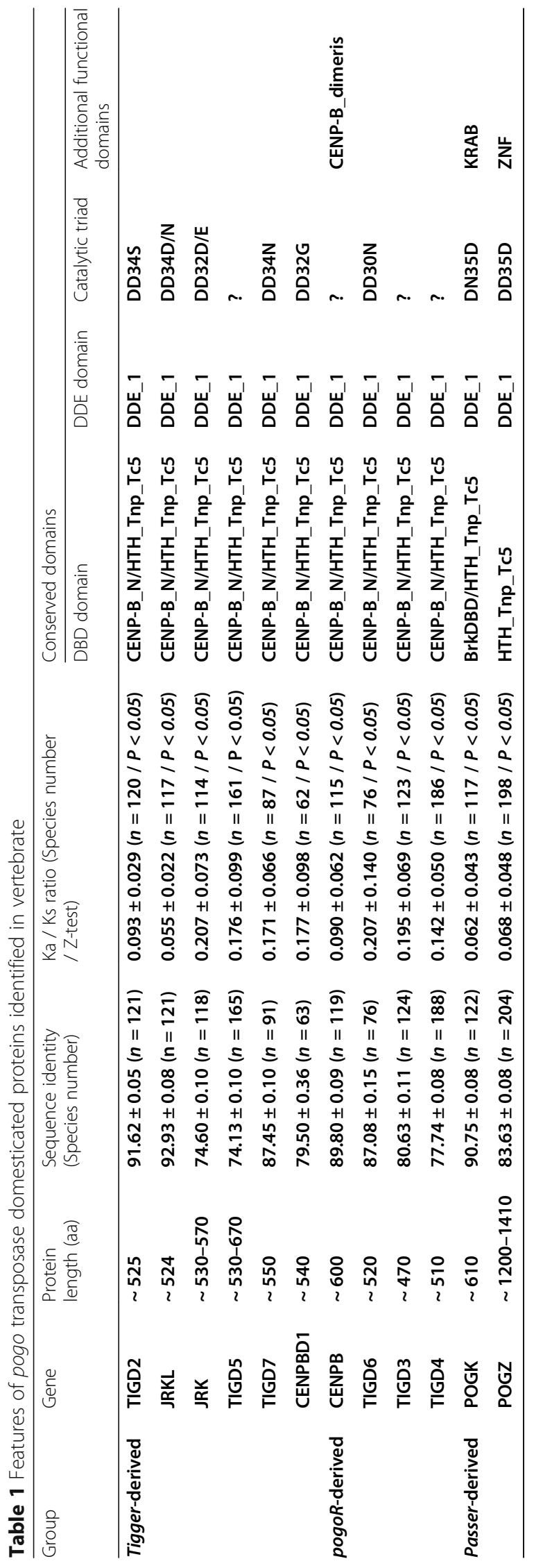


independent domestication events from different sources of pogo transposases. However, JRKL and TIGD2 appeared to emerge from a common transposase ancestor, and JRKL in the Monotremata seems to be the common ancestral gene of TIGD2 and JRKL, providing evidence for a co-domestication event of pogo transposons in vertebrates (Fig. 5b, Additional file 6: Fig. S4 and Additional file 7: Fig. S5).

\section{Structural conservation of pogo transposase domesticated genes}

Examination of the domain architecture of these domesticated proteins compared with pogo transposases revealed that most domesticated genes are derived from the complete transposase genes and show the same DBD and DDE domains found in pogo transposases; three of them (CENPB, POGK, and POGZ) are chimeric genes emerging from the fusion of entire transposase genes with additional functional domains (Fig. 6, Additional file 5: Table S2 and Additional file 8: Fig. S6). CENPB obtained an additional domain of dimerization in the Cterminal region, while POGK and POGZ obtained a Kruppel-associated box (KRAB) and zinc finger (ZNF) domain near the $\mathrm{N}$-terminus, respectively (Fig. 6, Additional file 5: Table S2 and Additional file 8: Fig. S6A-6C). Seven to nine ZNF finger motifs scattered in the $\mathrm{N}$-terminal of POGZs were identified (Additional file 8: Fig. S6C), which are now recognized to bind DNA, RNA, protein, and/or lipid substrates [54]. The DDE domain has been fully retained in all domesticated genes. The triad signatures of the DDE domain are well conserved in the JRK, JRKL, and POGZ sequences, and partially conserved in CENPBD1, TIGD2, TIGD6,
TIGD7, and POGK, but are not recognizable in CENPB, TIGD3, TIGD4, and TIGD5; Most domesticated genes harbor two type DBD motifs represented by CENP-B_N and HTH_Tnp_Tc5 in the N-terminal: POGKs are by BrkDBD and HTH_Tnp_Tc5 DBD motifs, while POGZs harbor only one DBD motif (HTH_Tnp_Tc5; Fig. 6, Additional file 5: Table S2 and Additional file 8: Fig. S6).

\section{Discussion}

We report a comprehensive analysis of the evolutionary history of pogo transposons in eukaryotes and of their repeated domestication in vertebrates. Many domesticated genes derived from DNA transposases have been characterized previously [11], with only SETMAR [13] and CENPB [39] known to derive from the Tc1/mariner superfamily. JRK, JRKL, and TIGD1-7 are also known as domesticated from pogo transposases [39], however, their structure organization, origins and taxonomic distribution are largely unknown. Our results first point to the common domestication events of pogo transposons-as a superfamily of ITm transposons-in vertebrates, where 12 well-annotated genes (CENPBD1, JRK, JRKL, POGK, POGZ, and TIGD2-7) beyond CENPB [39] were predicted to have evolved from this family. They were domesticated at different times during the evolution of vertebrates, with some very ancient domestication events leading to TIGD3, TIGD4, TIGD5, and POGZ. These genes first emerged in lobe-finned fish or Amphibia, similar to RAG1 [14] and PBGD5 [55]. More recent domestication events of CENPB, JRK, JRKL, POGK, and TIGD7 occurred in the ancestor of Mammalia, prior to the divergence of Theria and Monotremata, and TIGD2 and TIGD6 emerged after the split of the

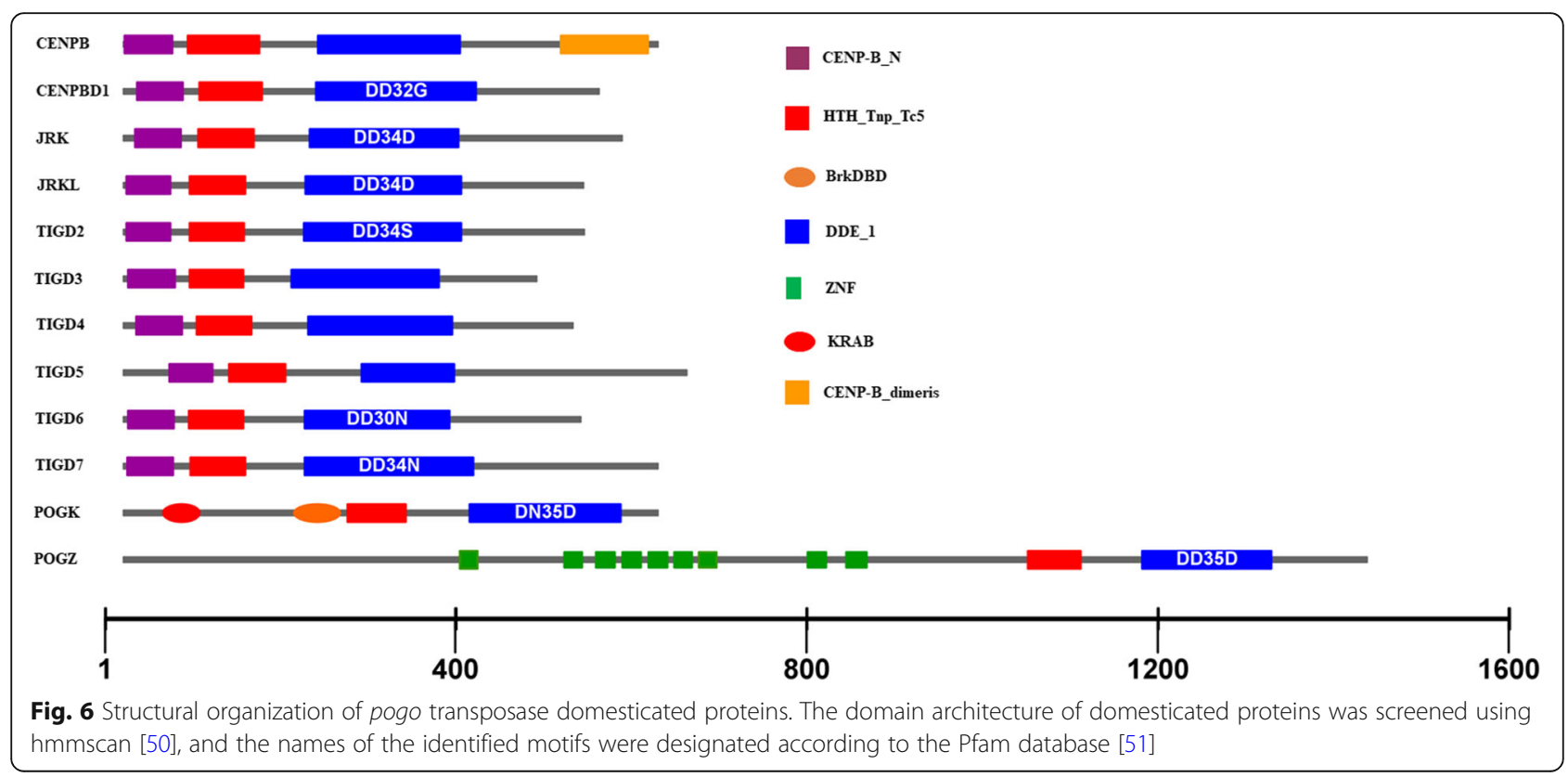


marsupials and eutheria, while the very recent domestication of CENPBD1 is present only in some mammalian lineages. Here, we clearly defined the putative origins of these domesticated genes for the first time. Our data suggests that only TIGD2, TIGD5, and TIGD7 originated from Tigger transposases, while TIGD3, TIGD4, and TIGD6 are derived from pogoR transposases. Furthermore, the phylogenetic tree we constructed revealed that most genes have emerged from different transposons and represent independent domestication events. However, our analysis also suggests that TIGD2 and JRKL seem to have originated from the same Tigger transposon, and might represent a co-domestication event similar to the one found in bony vertebrates (HARBI1 and NAIF1) [56] and Drosophila (DPLG7 and DPMG7) [57]. The functions of these genes seem to be related to DNA binding, and both POGZ and CENPB are believed to act in modulating chromatin structure $[40,58]$. It has been demonstrated that disruptions of POGZ are associated with autism [5961], while inactivation of JRK in mice result in epileptic seizures [62], and JRK was found to be overexpressed in cancers [63].

Our study provides a global overview of the evolutionary relationships among pogo, Tc1/mariner, Gambol, and Zator transposons and refines the classification of the ITm group. Zator has been found to derive from TP36 insertion sequences in bacteria [28], and suggested to be a separate superfamily being distantly related to the Tc1/mariner and bacterial IS630 elements. However, both of pogo and Gambol once were classified as the families of Tc1/mariner transposons [25, 26, 47]. Gambol was identified in African malaria mosquito, and is characterized by a typical DD34E catalytic triad and TA TSDs. However, it was found to form a distinct group separated from DD34E/Tc1 according to previous phylogenetic analyses [47]. Here, our phylogenetic analysis including more IS630 representative sequences from the ISfinder database demonstrated that pogo, Gambol, and Tc1/mariner form well-supported monophyletic clades. We thus conclude that they are separate superfamilies that may have originated from different clades of bacterial IS630 TEs and evolve independently, like Zator.

Our findings also suggest that pogo transposons might display the widest taxonomic distribution compared with the other Tc1/mariner superfamilies, as well as other superfamilies of DNA transposons [18]. The DD34D/ mariner and $D D 34 E / T c 1$ families have been examined in detail. DD34D/mariner was once regarded as the most widely distributed family of transposons, represented in diverse taxa such as fungi, ciliates, rotifers, insects, nematodes, plants, fish, and mammals [18]. However, we found that the pogo transposons are even more widely distributed in nature, with an expansion spanning across all kingdoms of eukaryotes. In addition, the taxonomic distribution of pogo transposons were underestimated since the ancient elements with truncated TIRs were excluded from our analysis. Our analysis also indicates that this superfamily has undergone a massive amplification in fungi with a wide taxonomic distribution, and is widespread in animals, where pogo transposons invaded almost all phyla of invertebrates and most classes of vertebrates, suggesting that the pogo transposons have played important roles in shaping the evolution of fungal and animal genomes. However, they did not accumulate significantly in land plants-being found in only 23 species-indicating a restricted influence of this superfamily on plant genome evolution.

We discovered that the pogo superfamily displays an unexpected level of diversity at the family and clade levels with significant variations in structural organization. Compared with other DNA transposons, pogo might represent the highest such diversity, with at least six distinct families defined (Tigger, pogoR, Lemi, Mover, Passer, and Fot/Fot-like). Furthermore, some families also display intra-group diversity and contain distinctive multiple clades, such as Fot/Fot-like, where at least four distinct clades (FotA-D) with well-supported bootstraps $(>=93 \%)$ were identified. Compared with the classic structures of Tc1/mariner transposons [16, 25], we found that pogo transposons show significant structural variations, including the transposon hallmarks of TSD and TIR sequences and the transposase domains DBD and DDE. Two new types of TSD (TAAA and TAA) have now been identified in pogo transposons in addition to the general TSD type (TA) of the Tc1/mariner superfamily. Significant variability (DD29-56D) of the number of amino acids between the last two residues of the triad signatures of the DDE domain was observed across different families, or between different clades of the same family, which is unique compared with most other families of Tc1/mariner.

\section{Conclusions}

This is the first report to systematically revealing the evolutionary profiles of the pogo transposons, which was defined as a new superfamily of the ITm group and displays a high family diversity and very wide taxonomic distribution in nature, with a massive amplification in fungi and animals, but narrow distribution in land plants. Furthermore, we also provided evidence to support that pogo superfamily has been domesticated repeatedly in vertebrates, over 10 functional genes were deduced as originating from this superfamily. Ten of these originate from different sources of pogo transposases and represent independent domestication events, while two of them seem to have been co-domesticated. This study expands our understanding of the evolution 
of ITm transposons, and these data suggest that the pogo superfamily contributes significantly to diversifying and shaping the genomes of fungi and animals, as well as functional genes in vertebrates.

\section{Materials and methods}

\section{Transposons mining}

To determine the distributions of pogo transposons, 2612 sequences of Tc1/mariner transposons were downloaded from the RepBase (20181026) database [64] and combined with six sequences of pogo-like transposons from different teleost species, including cod (Gadus morhua), medaka (Oryzias latipes), stickleback (Gasterosteus aculeatus), tetraodon (Tetraodon nigroviridis), tilapia (Oreochromis niloticus), and zebrafish (Danio rerio), which were identified in our previous studies $[9,38]$, to generate 964 transposase sequences (>300 aa); 302 sequences were identified as pogo transposases based on the phylogenetic analysis according to the references [ 9 , 38]. Then, these pogo transposase sequences were used as queries to search against the available organism genomes, including prokaryotes (bacteria and archaea) and eukaryota, which comprise plants (red algae, green algae, and land plants), Chromista (Stramenopiles, Alveolates, and Rhizaria), protozoa (Amoebozoa, Excavata, Ichthyosporea, and Choanoflagellata), fungi, and animals, at the database of the National Center for Biotechnology Information (NCBI) by using TBlastN with a cutoff value of $1^{\mathrm{e}-100}$. The new sequences identified were then used as queries to identify more elements. The top 10 nonoverlapping hits were extracted along with $2 \mathrm{~kb}$ of flanking sequences, and aligned using the MAFFT program [65] to identify the transposon boundaries manually. Elements with two detectable TIRs and TSDs of DNA transposons, or elements coding for transposases of at least 300 aa with one TIR and TSD, are referred to as transposons, the truncated elements with only one TIR coding for transposases of less than 300 aa or undetectable TIRs were discarded, which may be ancient invasion copies. Then, these representative sequences were subjected to BLAST analysis of each host genome to estimate copy numbers. All BLAST hits $>1000 \mathrm{bp}$ in size and $>80 \%$ identity were used to calculate copy numbers. In addition, the transposons with very few copies $(<3)$ in genomes, which may be false positives due to the sequence contamination, the flanking sequences of these transposons were further mapped to the host genome or the closely related species genomes, the un-mapping transposons were excluded for the analysis.

\section{Domesticated gene mining}

The domesticated genes of these transposons were identified in the vertebrate species only with the Reference and Representative genomes deposited in RefSeq
Representative Genome Database of NCBI, where the genomes in this database were well assembled and are among the best quality genomes available at NCBI. The domesticated genes were identified using the representative pogo transposases from different subfamilies as queries search against the NCBI genome databases available by using TBlastN with a cutoff value of $1^{\mathrm{e}-100}$. Here, to discriminate between transposons sequences from domesticated genes, TBlastN was used to align each sequence with $2 \mathrm{~kb}$ flanking sequences on the host genome to detect potential TIR and TSD sequences. When TIRs and TSDs were found on both sides or one side, the sequence was considered to be a transposon, while sequences flanked by no TIR or TSD sequences were considered to be putatively domesticated genes. The structure of each domesticated gene sequence obtained using the TblastN program was predicted initially using GENSCAN (http://hollywood.mit.edu/GENSCAN. html) and refined by alignment with orthologous genes. Sequences used as vectors were removed, and in case of isoform proteins, only one sequence was selected. The remaining sequences were then submitted for classification and phylogenetic analysis. The average sequence identity of proteins was estimated by the multiple sequence alignment program (emma) embedded in EMBOSS (http://www.bioinformatics.nl/emboss-explorer/). It is not easy to distinguish transposons from transposon-derived genes in those genomes where large amounts of related and recently active transposons are found; therefore, we applied a stringent standard to filter out ambiguous domesticated genes. Gene clades with a low average sequence identity of proteins $(<70 \%)$, very narrow taxonomic distribution (fewer than five species), or multiple copies $(>3)$ in genomes were excluded from the domestication analysis.

\section{Domain architecture and phylogenetic analysis}

The protein domains were identified using hidden Markov Models with the online hmmscan web server (https://www.ebi.ac.uk/Tools/hmmer/search/hmmscan)

[50]. The ZFN sequence was predicted using an online web server (http://zf.princeton.edu/logoMain.php). To define the phylogenetic position of pogo transposons accurately, all bacteria IS630 transposase sequences were retrieved from ISfinder database [41] and the DDE domains were extracted by using hmmalign program in HMMER (v3.3, http://hmmer.org/). Then, they (121 sequences) were aligned with MAFFT program [66] and submitted for classification by using the maximum likelihood method within IQ-TREE (v. 1.6.1) [48]. The bacteria IS256 transposase was used as outgroup. Then, the representative sequences of IS630 transposases from each clade and other unclassified sequences, Tc1/mariner known families transposases [21-23, 26, 42-47], and 
Zatror transposases [28] were jointed with the pogo transposases to infer the phylogenetic tree based on the multiple amino acid alignment of the conserved DDE domain by using the maximum likelihood method within IQ-TREE [48]. The best-fit model was selected by ModelFinder embedded in IQ-TREE [48], and the reliability of maximum likelihood trees was estimated by using the ultrafast bootstrap approach with 1000 replicates. The evolutionary histories of the domesticated proteins of $D D \times D$ transposases were inferred based on the alignments of DDE domains by using the IQ-TREE program as well [48], but the IS630 family was used as an outgroup.

\section{Codon substitution pattern and statistical analysis}

Coding sequences for domesticated genes of pogo transposases in vertebrates were aligned using ClustalW embedded in MEGA 7.0.26 [67], and the number of nonsynonymous substitutions per nonsynonymous site (Ka) and the number of synonymous substitutions per synonymous site (Ks) were estimated using the NeiGojobori method. The codon-based tests of selection analyses were conducted in MEGA with a $\mathrm{Z}$ test by calculating the substitution ratio of $\mathrm{Ka} / \mathrm{Ks}$ [53]. Then, the $\mathrm{Ka} / \mathrm{Ks}$ ratios were calculated to assess selection pressure using $\mathrm{Z}$ tests. The variance of the difference was computed using the bootstrap method (100 replicates). Orthologous sequences with a $\mathrm{Ka} / \mathrm{Ks}$ value of $<1$ (Z-test, $P<0.05)$ were defined as having been under purifying selection.

\section{Supplementary information}

Supplementary information accompanies this paper at https://doi.org/10. 1186/s13100-020-00220-0.

Additional file 1: Fig. S1. 15630 transposase classification. The phylogenetic tree was inferred using the maximum likelihood method with the IQ-Tree program, as described in the Materials and Methods. IS256 transposase was used as an outgroup.

Additional file 2: Fig. S2. Uncollapsed phylogenetic tree of pogo transposases. The phylogenetic tree was inferred using the maximum likelihood method with the IQ-Tree program, as described in the Materials and Methods.

Additional file 3: Table S1. Distribution of pogo transposons. List of all species/organism containing pogo transposon. For each species, the following information is provided: classification information of species, transposon Name ID, structural characteristics of representative transposon, including copy number (Blast hits $>1000$ bp and identity $>$ $80 \%)$, transposon ( $\mathrm{Tn}$ ) length, transposase (Tpase) length (> $300 \mathrm{aa})$, TIR length, TIR end motif, TIR sequence, TSD, domains of transposase, and triad signature of the DDE domain, and genome coordinate and sequence of representative transposon.

Additional file 4: Fig. S3. The intra-group classification and distributions of Lemi and Fot transposons in fungi and land plants. (A) Subphylogenetic tree of Lemi transposases constructed using the maximum likelihood method. (B) Subphylogenetic tree of Fot transposases constructed using the maximum likelihood method. The number of species/ organisms containing Lemi and Fot elements for each clade is given in brackets.
Additional file 5: Table S2. Distribution of pogo transposon domesticated proteins.

Additional file 6: Fig. S4. Uncollapsed phylogenetic tree of pogo transposases domesticated proteins. The tree was inferred using the maximum likelihood method with the IQ-Tree program, as described in the Materials and Methods. The DD35E/IS630 family was used as an outgroup.

Additional file 7: Fig. S5. Uncollapsed phylogenetic tree of pogo transposons domesticated proteins including pogo transposases. The tree was inferred using the maximum likelihood method in the IQ-Tree program, as described in the Materials and Methods. The DD35E/IS630 family was used as an outgroup. The pseudogenes (PS) of JRK, TIGD3, TIGD5, TIGD7, and POGZ are labeled as JRK PS, TIGD3 PS, TIGD5 PS, TIGD7 PS, and POGZ PS, respectively.

Additional file 8: Fig. S6. Alignment of pogo transposase domesticated proteins.

\section{Acknowledgments}

We thank Zoltán Ivics for a critical reading of the manuscript.

\section{Authors' contributions}

C.S. conceived the project; B.G. participated in its design; B.G., Y.W., D.M., D.S., S.W., C.C., X. W and W.Z. performed the data mining and analyses; and C.S. and B.G. wrote and revised the manuscript with input from all authors. The author(s) read and approved the final manuscript.

\section{Funding}

This research was supported by grants from the National Genetically Modified Organisms Breeding Major Projects program (2018ZX08010-08B), the National Natural Science Foundation of China (31671313), the Priority Academic Program Development of Jiangsu Higher Education Institutions, and the High-end Talent Support Program of Yangzhou University.

Availability of data and materials

All data needed to evaluate the conclusions in this paper are present either in the main text or the Supporting information.

Ethics approval and consent to participate

Not applicable.

Consent for publication

Not applicable.

\section{Competing interests}

The authors declare that have no competing interests.

Received: 3 March 2020 Accepted: 26 June 2020

Published online: 08 July 2020

\section{References}

1. Kleckner N. Prokaryotic transposable elements. Annu Rev Genet. 1981;15: 341-404.

2. Finnegan DJ. Transposable elements in eukaryotes. Int Review Cytol. 1985; 93:281-326

3. Bourque G, Burns KH, Gehring M, Gorbunova V, Seluanov A, Hammell M, Imbeault M, Izsvák Z, Levin HL, Macfarlan TS, Mager DL. Ten things you should know about transposable elements. Genome Biol. 2018;19(1):199.

4. Feschotte C, Pritham EJ. DNA transposons and the evolution of eukaryotic genomes. Annu Rev Genet. 2007;41:331-68.

5. He S, Corneloup A, Guynet C, Lavatine L, Caumont-Sarcos A, Siguier P, et al. The IS200/IS605 family and "Peel and paste" single-strand transposition mechanism. Microbiol Spectr. 2015.

6. Kazazian HH. Mobile elements: drivers of genome evolution. Science. 2004; 303(5664):1626-32.

7. Sinzelle L, Izsvak Z, Ivics Z. Molecular domestication of transposable elements: from detrimental parasites to useful host genes. Cell Mol Life Sci. 2009;66(6):1073-93. 
8. Chalopin D, Naville M, Plard F, Galiana D, Volff J-N. Comparative analysis of transposable elements highlights Mobilome diversity and evolution in vertebrates. Genome Biol Evol. 2015;7:567-80.

9. Gao B, Shen D, Xue S, Chen C, Cui H, Song C. The contribution of transposable elements to size variations between four teleost genomes. Mob DNA. 2016;7(1):4.

10. Platt RN, Vandewege MW, Ray DA. Mammalian transposable elements and their impacts on genome evolution. Chromosom Res. 2018;26(1-2):25-43.

11. Alzohairy AM, Gyulai G, Jansen RK, Bahieldin A. Transposable elements domesticated and neofunctionalized by eukaryotic genomes. Plasmid. 2013; 69(1):1-5.

12. Majumdar S, Singh A, Rio DC. The human THAP9 gene encodes an active Pelement DNA transposase. Science. 2013;339(6118):446-8.

13. Liu D, Bischerour J, Siddique A, Buisine N, Bigot Y, Chalmers R. The human SETMAR protein preserves most of the activities of the ancestral Hsmar1 transposase. Mol Cell Biol. 2007;27(3):1125-32.

14. Huang S, Tao X, Yuan S, Zhang Y, Li P, Beilinson HA, Zhang Y, Yu W, Pontarotti $P$, Escriva $H$, Le Petillon $Y$. Discovery of an active RAG transposon illuminates the origins of $V(D) J$ recombination. Cell. 2016;166(1):102-14.

15. Henssen AG, Henaff $E$, Jiang E, Eisenberg AR, Carson JR, Villasante CM, Ray M, Still E, Burns M, Gandara J, Feschotte C. Genomic DNA transposition induced by human PGBD5. Elife. 2015:4:e10565.

16. Plasterk RH, Izsvák Z, Ivics Z. Resident aliens: the Tc1/mariner superfamily of transposable elements. Trends Genet. 1999;15(8):326-32.

17. Jacobson JW, Medhora MM, Hartl DL. Molecular structure of a somatically unstable transposable element in Drosophila. Proc Natl Acad Sci. 1986;83: 8684-8.

18. Muñoz-López M, García-Pérez JL. DNA transposons: nature and applications in genomics. Curr Genomics. 2010;11:115-28.

19. Zhang HH, Shen $Y H$, Xiong XM, Han MJ, Zhang XG. Identification and evolutionary history of the DD41D transposons in insects. Genes Genomics. 2016:38:109-17.

20. Nguyen DH, Hermann D, Caruso A, Tastard E, Marchand J, Rouault JD, et al. First evidence of mariner-like transposons in the genome of the marine microalga Amphora acutiuscula (Bacillariophyta). Protist. 2014;165:730-44.

21. Bouallègue M, Filée J, Kharrat I, Mezghani-Khemakhem M, Rouault JD, Makni M, Capy P. Diversity and evolution of mariner-like elements in aphid genomes. BMC Genomics. 2017;18(1):494.

22. Sang Y, Gao B, Diaby M, Zong W, Chen C, Shen D, et al. Incomer, a DD36E family of Tc1/mariner transposons newly discovered in animals. Mob DNA 2019;10:45.

23. Zong W, Gao B, Diaby M, Shen D, Wang S, Wang Y, Sang Y, Chen C, Wang $X$, Song C. Traveler, a new DD35E family of TC1/mariner transposons, invaded vertebrates very recently. Genome Biol Evol. 2020;12(3):66-76.

24. Doak TG, Doerder FP, Jahn CL, Herrick G. A proposed superfamily of transposase genes: transposon-like elements in ciliated protozoa and a common" D35E" motif. Proc Natl Acad Sci. 1994:91(3):942-6.

25. Tellier M, Bouuaert CC, Chalmers R. Mariner and the ITm superfamily of transposons. Mobile DNA III. 2015:753-72.

26. Shao H, Tu Z. Expanding the diversity of the IS630-TC1-mariner superfamily: discovery of a unique DD37E transposon and reclassification of the DD37D and DD39D transposons. Genetics. 2001;159(3):1103-15.

27. Kojima KK. Structural and sequence diversity of eukaryotic transposable elements. Genes Genet Syst. 2018:18-00024.

28. Bao W, Jurka MG, Kapitonov W, Jurka J. New superfamilies of eukaryotic DNA transposons and their internal divisions. Mol Biol Evol. 2009;26(5):98393

29. Tudor M, Lobocka M, Goodell M, Pettitt J, O'Hare K. The pogo transposable element family of Drosophila melanogaster. Mol Gen Genet MGG. 1992; 232(1):126-34.

30. Smit AF, Riggs AD. Tiggers and DNA transposon fossils in the human genome. Proc Natl Acad Sci. 1996;93(4):1443-8.

31. Dufresne M, Lespinet O, Daboussi MJ, Hua-Van A. Genome-wide comparative analysis of pogo-like transposable elements in different Fusarium species. J Mol Evol. 2011;73(3-4):230-43.

32. Hey P, Robson G, Birch M, Bromley M. Characterisation of Aft1 a Fot1/Pogo type transposon of Aspergillus fumigatus. Fungal Genet Biol. 2008;45(2):11726.

33. Nyyssönen $E$, Amutan M, Enfield L, Stubbs J, Dunn-Coleman NS. The transposable element Tan1 of Aspergillus niger var. awamori, a new member of the Fot1 family. Mol Gen Genet MGG. 1996;253(1-2):50-6.
34. Levis C, Fortini D, Brygoo Y. Flipper, a mobile Fot1-like transposable element in Botrytis cinerea. Mol Gen Genet MGG. 1997;254(6):674-80.

35. Kachroo P, Leong SA, Chattoo BB. Pot2, an inverted repeat transposon from the rice blast fungus Magnaporthe grisea. Mol Gen Genet MGG. 1994;245(3): 339-48.

36. Daboussi MJ, Langin T, Brygoo Y. Fot1, a new family of fungal transposable elements. Mol Gen Genet MGG. 1992;232(1):12-6.

37. Feschotte $\mathrm{C}$, Mouches $\mathrm{C}$. Evidence that a family of miniature inverted-repeat transposable elements (MITEs) from the Arabidopsis thaliana genome has arisen from a pogo-like DNA transposon. Mol Biol Evol. 2000;17(5):730-7.

38. Gao B, Chen W, Shen D, Wang S, Chen C, Zhang L, et al. Characterization of autonomous families of Tc1/mariner transposons in neoteleost genomes. Mar Genomics. 2017:34:67-77.

39. Casola C, Hucks D, Feschotte C. Convergent domestication of pogo-like transposases into centromere-binding proteins in fission yeast and mammals. Mol Biol Evol. 2008;25(1):29-41.

40. Nozawa RS, Nagao K, Masuda HT, Iwasaki O, Hirota T, Nozaki N, Kimura H, Obuse C. Human POGZ modulates dissociation of HP1a from mitotic chromosome arms through Aurora B activation. Nat Cell Biol. 2010;12(7): 719-27.

41. Siguier P, Pérochon J, Lestrade L, Mahillon J, Chandler M. ISfinder: the reference centre for bacterial insertion sequences. Nucleic Acids Res. 2006; 34(suppl_1):D32-6.

42. Zhang HH, Li GY, Xiong XM, Han MJ, Zhang XG, Dai FY. TRT, a vertebrate and protozoan Tc1-like transposon: current activity and horizontal transfer. Genome Biol Evol. 2016;8(9):2994-3005.

43. Clark KJ, Carlson DF, Leaver MJ, Foster LK, Fahrenkrug SC. Passport, a native Tc1 transposon from flatfish, is functionally active in vertebrate cells. Nucleic Acids Res. 2009;37:1239-47.

44. Miskey C, Izsvák Z, Plasterk RH, Ivics Z. The Frog Prince: A reconstructed transposon from Rana pipiens with high transpositional activity in vertebrate cells. Nucleic Acids Res. 2003;31:6873-81.

45. Ke Z, Grossman GL, Cornel AJ, Collins FH. Quetzal: a transposon of the TC1 family in the mosquito Anopheles albimanus. Genetica. 1996:98(2):141-7.

46. Mátés L, Chuah MK, Belay E, Jerchow B, Manoj N, Acosta-Sanchez A, Grzela DP, Schmitt A, Becker K, Matrai J, Ma L. Molecular evolution of a novel hyperactive Sleeping Beauty transposase enables robust stable gene transfer in vertebrates. Nat Genet. 2009:41(6):753-61.

47. Coy MR, Tu Z. Gambol and TC1 are two distinct families of DD34E transposons: analysis of the Anopheles gambiae genome expands the diversity of the IS630-TC1-mariner superfamily. Insect Mol Biol. 2005;14(5): 537-46.

48. Nguyen LT, Schmidt HA, Von Haeseler A, Minh BQ. IQ-TREE: a fast and effective stochastic algorithm for estimating maximum-likelihood phylogenies. Mol Biol Evol. 2015;32(1):268-74.

49. Daboussi MJ, Daviere JM, Graziani S, Langin T. Evolution of the Fot1 transposons in the genus Fusarium: discontinuous distribution and epigenetic inactivation. Mol Biol Evol. 2002;19(4):510-20.

50. Finn RD, Clements J, Arndt W, Miller BL, Wheeler TJ, Schreiber F, Bateman A, Eddy SR. HMMER web server: 2015 update. Nucleic Acids Res. 2015;43(W1): W30-8

51. Finn RD, Coggill P, Eberhardt RY, Eddy SR, Mistry J, Mitchell AL, Potter SC, Punta M, Qureshi M, Sangrador-Vegas A, Salazar GA. The Pfam protein families database: towards a more sustainable future. Nucleic Acids Res. 2016:44(D1):D279-85.

52. Wicker T, Sabot F, Hua-Van A, Bennetzen JL, Capy P, Chalhoub B, Flavell A, Leroy $P$, Morgante $M$, Panaud $O$, Paux E. A universal classification of eukaryotic transposable elements implemented in Repbase. Nat Rev Genet. 2008;9(5):414.

53. Yang Z. PAML: a program package for phylogenetic analysis by maximum likelihood. Comput Appl Biosci. 1997;13(5):555-6.

54. Laity JH, Lee BM, Wright PE. Zinc finger proteins: new insights into structural and functional diversity. Curr Opin Struct Biol. 2001;11(1):39-46.

55. Bouallegue M, Rouault JD, Hua-Van A, Makni M, Capy P. Molecular evolution of piggyBac superfamily: from selfishness to domestication. Genome Biol Evol. 2017:9(2):323-39.

56. Sinzelle L, Kapitonov W, Grzela DP, Jursch T, Jurka J, Izsvák Z, Ivics Z. Transposition of a reconstructed Harbinger element in human cells and functional homology with two transposon-derived cellular genes. Proc Natl Acad Sci. 2008:105(12):4715-20. 
57. Casola C, Lawing AM, Betrán E, Feschotte C. PIF-like transposons are common in Drosophila and have been repeatedly domesticated to generate new host genes. Mol Biol Evol. 2007;24(8):1872-88.

58. Okada T, Ohzeki Jl, Nakano M, Yoda K, Brinkley WR, Larionov V, Masumoto H. CENP-B controls centromere formation depending on the chromatin context. Cell. 2007;131(7):1287-300,

59. Study TD, Fitzgerald TW, Gerety SS, Jones WD, van Kogelenberg M, King DA, McRae J, Morley KI, Parthiban V, Al-Turki S, Ambridge K. Largescale discovery of novel genetic causes of developmental disorders. Nature. 2015;519(7542): 223-8.

60. Stessman HA, Willemsen MH, Fenckova M, Penn O, Hoischen A, Xiong B, Wang T, Hoekzema K, Vives L, Vogel I, Brunner HG. Disruption of POGZ is associated with intellectual disability and autism spectrum disorders. Am J Hum Genet. 2016;98(3):541-52.

61. White J, Beck CR, Harel T, Posey JE, Jhangiani SN, Tang S, Farwell KD, Powis Z, Mendelsohn NJ, Baker JA, Pollack L. POGZ truncating alleles cause syndromic intellectual disability. Genome Med. 2016;8(1):1-1.

62. Toth M, Grimsby J, Buzsaki G, Donovan GP. Epileptic seizures caused by inactivation of a novel gene, jerky, related to centromere binding protein-B in transgenic mice. Nat Genet. 1995;11(1):71-5.

63. Pangon L, Ng I, Giry-Laterriere M, Currey N, Morgan A, Benthani F, Tran PN Al-Sohaily S, Segelov E, Parker BL, Cowley MJ. JRK is a positive regulator of $\beta$-catenin transcriptional activity commonly overexpressed in colon, breast and ovarian cancer. Oncogene. 2016:35(22):2834-41.

64. Bao W, Kojima KK, Kohany O. Repbase Update, a database of repetitive elements in eukaryotic genomes. Mob DNA. 2015;6(1):11.

65. Thompson JD, Gibson TJ, Plewniak F, Jeanmougin F, Higgins DG. The CLUSTAL_X windows interface: flexible strategies for multiple sequence alignment aided by quality analysis tools. Nucleic Acids Res. 1997;25(24): 4876-82.

66. Katoh K, Standley DM. MAFFT multiple sequence alignment software version 7: improvements in performance and usability. Mol Biol Evol. 2013; 30(4):772-80.

67. Kumar S, Stecher G, Tamura K. MEGA7: molecular evolutionary genetics analysis version 7.0 for bigger datasets. Mol Biol Evol. 2016;33(7):1870-4.

\section{Publisher's Note}

Springer Nature remains neutral with regard to jurisdictional claims in published maps and institutional affiliations.

Ready to submit your research? Choose BMC and benefit from:

- fast, convenient online submission

- thorough peer review by experienced researchers in your field

- rapid publication on acceptance

- support for research data, including large and complex data types

- gold Open Access which fosters wider collaboration and increased citations

- maximum visibility for your research: over $100 \mathrm{M}$ website views per year

At $\mathrm{BMC}$, research is always in progress.

Learn more biomedcentral.com/submissions 